\title{
Polimorfismo (+3954C>T) del Gen IL-1B y su Asociación con la Resorción Radicular Apical Externa Post-Tratamiento Ortodóntico
}

\author{
IL-1B (+3954C >T) Gene Polymorphism and its Association \\ with External Root Resorption after Orthodontic Treatment
}

Fanny Lince Vides"; Julio De La Ossa Salcedo**; Rubi Hernandez Tirado*;
Yaleyvis Buelvas Montes ${ }^{* * *}$; José Bustillo Arrieta ${ }^{* * *}$ \& Meisser Madera Anaya

LINCE, V. F.; DE LA OSSA, S. J.; HERNANDEZ, T. R.; BUELVAS, M. Y.; BUSTILLO, A. J. \& MADERA, A. M. Polimorfismo $(+3954$ C > T) del gen IL-1B y su asociación con la resorción radicular apical externa post-tratamiento ortodóntico. Int. J. Odontostomat., 10(2):243-248, 2016.

RESUMEN: El objetivo fue determinar la presencia del polimorfismo rs1143634 (+3954C>T) del gen Interleuquina 1 Beta (IL-1B) y su asociación con la resorción radicular apical externa (RRE) post-tratamiento ortodóntico. Se realizó un estudio piloto de individuos tratados con aparatología ortodontica, 13 (casos) presentaron RRE posterior al tratamiento ortodóntico y 22 (controles) estaban clínicamente sanos. A partir de muestras de células epiteliales de mucosa bucal se extrajo ADN y se genotipificó el polimorfismo rs1143634 (+3954C>T) del gen IL-1B mediante la reacción en cadena de la polimerasa y digestión del producto con la enzima de restricción Taql. Se estimaron las frecuencias alélicas y genotípicas del rs1143634; además, se evaluó la desviación del equilibrio de Hardy-Weinberg. Las frecuencias alélicas y genotípicas se compararon mediante la prueba de $c 2$ con razón de verosimilitud $(p<0,05)$. El promedio de edad de los participantes fue $28,1(\mathrm{DE}=11,5)$ años y el $68,6 \%$ era mujeres. Al comparar la distribución de los genotipos del polimorfismo IL-1B (+3954C>T) entre grupos no se encontró una diferencia estadísticamente significativa $(p=0,0926)$. Sin embargo, se observó una diferencia significativa en la distribución de alelos $(p=0,035)$, siendo el alelo T (alelo 2$)$ más prevalente en el grupo control. El polimorfismo IL-1B (+3954C>T) se encontró presente en la población de estudio. Aunque no existieron diferencias en la distribución de los genotipos que apoyara una asociación entre este polimorfismo y la RRE, si hubo una diferencia en la distribución de los alelos, sugiriendo que el alelo T posiblemente actúa como factor protector contra el desarrollo de la RRE.

PALABRAS CLAVE: reabsorción radicular externa, reabsorción apical, interleuquina 1 beta.

\section{INTRODUCCIÓN}

En la actualidad el tratamiento de ortodoncia es uno de los servicios especializados más solicitados en la práctica odontológica, debido a la capacidad que tiene de proporcionar funcionalidad oclusal y estética facial. Durante y luego de este tratamiento comúnmente se puede observar un remodelado apical de los dientes, el cual es considerado como una respuesta fisio- lógica adaptativa; sin embargo algunas veces por causas aún desconocidas se puede presentar la reabsorción radicular externa (RRE), entidad patológica asociada a un proceso de inflamación crónico en donde existe una pérdida mayor de $2 \mathrm{~mm}$ de cemento y dentina en la parte externa de la raíz (Ahangari et al., 2010; Diouf et al., 2015).

\footnotetext{
Odontóloga, Especialista en Ortodoncia, Magister en Bioquímica Clínica, Docente, Facultad de Odontología, Universidad de Cartagena, Cartagena, Colombia.

* Odontólogo, Especialista en Ortodoncia, Universidad de Cartagena, Cartagena, Colombia.

*** Bióloga con énfasis en Biotecnología; Magister en Microbiología, Docente, Facultad de Medicina, Universidad de Cartagena, Cartagena, Colombia.

Odontólogo, Especialista en Ortodoncia, Magister en Estadística Aplicada, Docente, Facultad de Odontología, Universidad de Cartagena, Cartagena, Colombia.

***** Odontólogo, Magister en Epidemiología Clínica, Magister en Bioquímica, Investigador del grupo GISPOUC, Facultad de Odontología, Universidad de Cartagena, Cartagena, Colombia.
} 
La RRE es multifactorial implicando tanto factores intrínsecos como extrínsecos (Bansal et al., 2015); se ha sugiriendo que su etiología durante el tratamiento de ortodoncia es compleja, involucrando la susceptibilidad genética, consumo de medicamentos, tabaquismo, hormonas, edad, antecedentes familiares y personales, tipo de aparatología ortodóntica, magnitud, dirección y duración de las fuerzas ejercidas durante movimientos dentales (Baysal et al., 2010; Gonzales et al., 2011; Villa et al., 2005); sin embargo, ninguno de estos factores ha generado una evidencia suficientemente sólida; por lo cual en la última década se han evaluado posibles asociaciones entre la susceptibilidad de RRE y biomarcadores genéticos (Al-Qawasmi et al., 2003a; Sharab et al., 2015).

Entre estos biomarcadores genéticos se ha sugerido que el gen IL-1B está relacionado con la RRE (Bastos et al., 2015; Bastos-Lages et al., 2009); este gen codifica a la citoquina IL-1, con dos isoformas (IL1a e IL-1b) capaces de estimular la diferenciación de los osteoclastos; específicamente se ha sugerido que la concentración de IL-1B en el fluido crevicular y en el tejido gingival es notablemente más alta durante el tratamiento ortodontico, lo que aumenta el riesgo de reabsorción radicular en los incisivos superiores (Ahangari et al.).

El objetivo de este trabajo fue determinar la presencia del polimorfismo rs1143634 (+3954C > T) del gen IL-1B y su asociación con la RRE post-tratamiento ortodóntico en individuos atendidos en las Clínicas Odontológicas de la Universidad de Cartagena.

\section{MATERIAL Y MÉTODO}

Tipo de estudio. Piloto de casos y controles, en donde los participantes recibieron tratamiento ortodóntico entre febrero del 2012 y mayo del 2014 en el postgrado de Ortodoncia de la Universidad de Cartagena, Colombia. Este estudio fue aprobado por el comité de ética de la Universidad de Cartagena.

Participantes. La muestra fueron 35 individuos, 13 participantes hicieron parte del grupo de casos, presentando RRE posterior al tratamiento y 22 fueron incluidos en el grupo control, los cuales no desarrollaron RRE al término de la ortodoncia. Para la selección de los participantes se tuvieron como criterios de inclusión: pacientes sin compromiso sistémico y/o discapacidad física o mental, con dentición permanente y formación radicular completa, sin previo tratamiento de ortodoncia. Asimismo se excluyeron aquellos individuos con historia de inflamación crónica de la pulpa $y$ tejidos periodontales, con antecedentes de traumas dentoalveolares, que estuvieran consumiendo algún tipo de medicamentos como Ibuprofeno y corticoides, fumadores y con hábitos nocivos como empuje lingual. Todos los participantes autorizaron su participación voluntaria mediante la firma del consentimiento informado.

Procedimientos. A todos los participantes se les realizó una radiografía panorámica (RX Panorámico Orto pantógrafo Kodak 8000c) antes y luego de dos años de tener el tratamiento de ortodoncia, en la primera radiografía se verificó el estado de las raíces dentales de los participantes para saber si cumplían con las criterios de inclusión; en la segunda se evaluó la presencia de RRE,identificando a aquellos dientes que mostraran una perdida igual o superior a $2 \mathrm{~mm}$, a estos se les tomó una radiografía periapical (RX ORIX 70) usando una reglilla milimetrada adosada a la película, mediante la técnica de paralelismo "66kv, 7.5 MA y $0.10 \mathrm{~s}$ ", con posicionador (RINN) y bloque de mordida anterior (BITE BLOCK XCP: 54-086). Durante la medición radicular se tomó como referencia anatómica la línea amelocementaria, proyectada sobre el canal radicular hasta el ápice. Estos procedimientos fueron realizados por un profesional previamente calibrado y estandarizado con un Kappan Cohen >0,80.

Obtención de las muestras de células epiteliales. Se tomó una muestra de células epiteliales de la mucosa bucal, cada individuo se enjuagó la boca durante 1 min con $5 \mathrm{~mL}$ de solución de glucosa al $3 \%$. Luego con un hisopo estéril se raspó suavemente la mucosa oral, después la punta de este se sumergió en tubos estériles que contenían $3 \mathrm{~mL}$ de solución salina, inmediatamente este tubo era transportado en hielo al Laboratorio de Microbiología de la Universidad de Cartagena para posterior análisis.

Extracción de ADN. La suspensión de células epiteliales se centrifugó a 2000 rpm durante $10 \mathrm{~min}$, el sobrenadante fue descartado y el pellet de células se resuspendió en $250 u$ ul del reactivo Ribozol (AMRESCO). El lisado fue transferido a un tubo eppendorf de $1,5 \mathrm{~mL}$, se incubó durante $5 \mathrm{~min}$ y se adicionó $50 \mathrm{~mL}$ de cloroformo, vortex por $30 \mathrm{~s}$ e incubación a temperatura ambiente durante $10 \mathrm{~min}$. Después las muestras se centrifugaron a $12.000 \mathrm{rpm}$ durante $15 \min$ a $4{ }^{\circ} \mathrm{C}$. La fase acuosa superior fue descartada y se adicionó $75 \mathrm{~mL}$ de etanol al $100 \%$. 


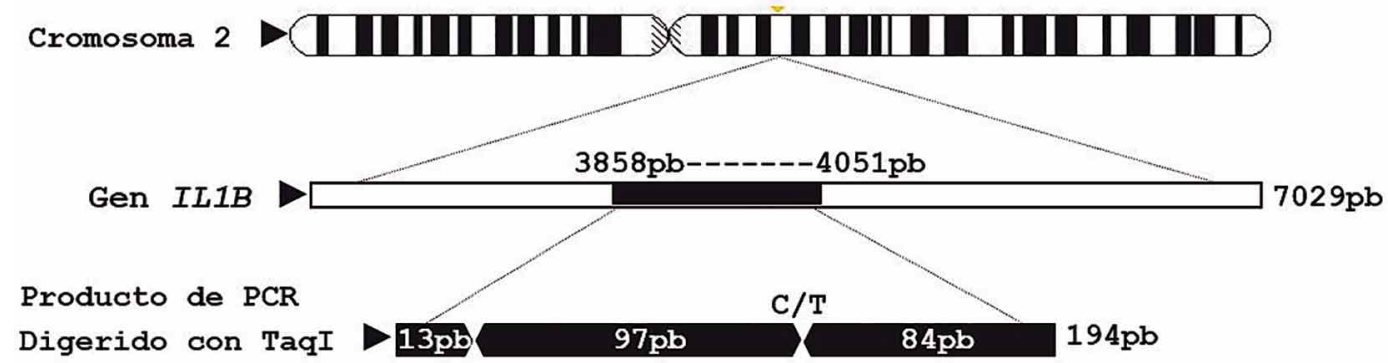

Fig. 1. Posición del gen IL-1B. Se observa posición del gen IL-1B en el brazo largo del cromosoma 2 y ubicación del polimorfismo $(\mathrm{C} / \mathrm{T})$, así como los sitios de corte de la enzima Taql en el fragmento amplificado por PCR.

Se mezcló por inversión y se incubó por 3 min. Luego se centrifugó a $4000 \mathrm{rpm}$ durante $5 \mathrm{~min}$ a $4{ }^{\circ} \mathrm{C}$ y el sobrenadante fue removido. El ADN fue lavado dos veces con $250 \mathrm{~mL}$ de solución $0,1 \mathrm{M}$ de trisodiumcitrate y $10 \%$ etanol. Durante cada lavado, se permitió que el ADN sedimentara durante 30 min haciendo vortex cada 5 min. Luego se centrifugó a 2.000 rpm por 5 min a $4{ }^{\circ} \mathrm{C}$ y se lavó con $100 \mathrm{~mL}$ de etanol $75 \%$. Finalmente se centrifugó a $9.000 \mathrm{rpm}$ durante $10 \mathrm{~min}$ y se descartó cuidadosamente el sobrenadante, resuspendiendo el ADN con $50 \mathrm{~mL}$ de agua libre de DNasas. La calidad del ADN extraído se determinó mediante espectrofotometría en el equipo NanoDrop 2000 UV-Vis Spectrophotometer (Thermo Scientific).

\section{Expresión génica mediante reacción en cadena de} polimerasa (PCR). Fue realizada una PCR para amplificar una región del gen IL-1B que porta el polimorfismo +3954 . Esta se realizó utilizando oligonucleótidos (5'-CTCAGGTGTCCTCGAAGAAATCAA-3'/5'-GCTTTTTTGCTGTGAGTCCCG-3) que amplifican un fragmento de $194 \mathrm{pb}$ del gen IL-1B. EI ADN fue amplificado en un volumen de reacción de $25 \mu \mathrm{L}$, que contenía $12,5 \mathrm{~mL}$ de la mezcla de PCR (PCR master Mix; Promega $($ ) ), 0,2 $\mu \mathrm{M}$ de cada primer y $5 \mu \mathrm{L}$ de ADN molde. La reacción se llevó a cabo en un termociclador (PerkinElmer $\circledast$ ) bajo las siguientes condiciones: un ciclo inicial de desnaturalización a 95 ${ }^{\circ} \mathrm{C}$ por 2 min, seguido por 38 ciclos de $95{ }^{\circ} \mathrm{C}$ por 1 $\min , 67^{\circ} \mathrm{C}$ por 1 min y $72^{\circ} \mathrm{C}$ por $1 \mathrm{~min}$, con un ciclo de extensión final a $72{ }^{\circ} \mathrm{C}$ por $8 \mathrm{~min}$. El producto de PCR fue digerido con 10 unidades por $30 \mu \mathrm{L}$ de reacción de Taql a $65^{\circ} \mathrm{C}$ durante $2 \mathrm{~h}$. Los productos resultantes son de $8 \mathrm{pb}+97 \mathrm{bp}$ para el alelo 1 y un único fragmento de $182 \mathrm{pb}$ para el alelo 2. En ambos casos, una banda constante de $12 \mathrm{pb}$ también fue generada, la cual sirve como un sitio de restricción de control (Fig. 1). Todos los productos fueron visualizados en gel de agarosa al $2 \%$ con bromuro de etidio $0,5 \mu \mathrm{g} / \mathrm{mL}$, me- diante un transiluminador de luz UV. Como gen de referencia se utilizó el gliceraldehido-3-fosfato deshidrogenasa (GAPDH).

Análisis estadístico. Se utilizó los programas estadísticos SPSS V19 (IBM ${ }^{\circledR}$ SPSS $®$ Statistics 20; IBM Corp., USA) y GraphPad Prism 6.0. Se estimaron las frecuencias alélicas y genotípicas; además se evaluó la desviación del equilibrio de Hardy-Weinberg de las frecuencias alélicas y se compararon entre cada individuo mediante la prueba de c2 con razón de verosimilitud para identificar la asociación genéticas. La estimación de Odd ratio (IC= $95 \%)$ se realizó mediante un modelo de regresión logística ajustado por las variables sexo y edad. Para todas las pruebas se asumió una probabilidad límite de decisión de 0,05.

\section{RESULTADOS}

La edad promedio de los participantes fue de $28,1(\mathrm{DE}=11,5)$ años, el $68,6 \%$ eran de sexo femenino y el $31,4 \%$ masculino; no se encontraron diferencias significativas en la distribución de estas variables según los grupos (Tabla I).

De las 35 muestras, seis no amplificaron el fragmento de GAPDH y por lo tanto fueron descartadas para realizar la determinación del polimorfismo del gen IL-1B; de los 29 (Casos=12; Controles= 17) productos restantes de PCR del gen IL1-B sometidos a digestión con la enzima Taql, 21 mostraron una sola banda de $90 \mathrm{pb}$ en el gel de agarosa al $2 \%$, indicando la digestión total del producto (homocigosis); 2 mostraron una banda de $194 \mathrm{pb}$ (producto no digerido); y 6 presentaron dos bandas, una de $194 \mathrm{pb}$ y otras de $90 \mathrm{pb}$, lo que indica la heterocigosis del polimorfismo (Fig. 2). 
LINCE, V. F.; DE LA OSSA, S. J.; HERNANDEZ, T. R.; BUELVAS, M. Y.; BUSTILLO, A. J. \& MADERA, A. M. Polimorfismo (+3954C>T) del gen IL-1B y su asociación con la resorción radicular apical externa post-tratamiento ortodóntico. Int. J. Odontostomat., 10(2):243-248, 2016.

Tabla I. Distribución de las características sociodemográficas de los participantes.

\begin{tabular}{llcccc}
\hline & & Total participantes & Casos & Controles & \multirow{2}{*}{ Valor $\boldsymbol{p}$} \\
\cline { 3 - 4 } & & $\mathrm{n}(\%)$ & $\mathrm{n}(\%)$ & $\mathrm{n}(\%)$ & \\
\hline Total muestra & & $35(100)$ & $13(37,1)$ & $22(62,9)$ & \\
Sexo & Masculino & $11(31,4)$ & $7(53,8)$ & $4(18,2)$ & \multirow{2}{*}{0,06} \\
& Femeninos & $24(68,6)$ & $6(46,2)$ & $18(81,8)$ & \\
Edad (años) & $<20$ & $10(28,6)$ & $2(15,4)$ & $8(36,4)$ & \multirow{2}{*}{0,26} \\
& $\geq 20$ & $25(71,4)$ & $11(84,6)$ & $14(63,6)$ & \\
\hline
\end{tabular}

*Estadísticamente significativo, prueba exacta de Fisher

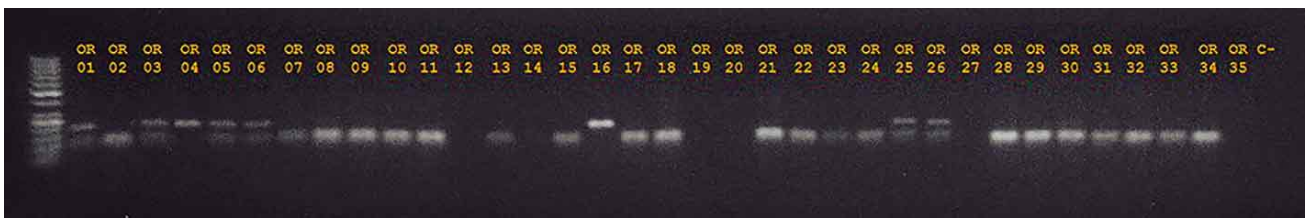

Fig. 2. Expresión génica del polimorfismo IL-1B. Electroforesis en gel de agarosa al $2 \%$ de los productos de PCR del gen IL-1B digerido con la enzima de restricción Taql. En el primer carril se encuentra el marcador de peso molecular, el tamaño del producto no digerido es de $194 \mathrm{pb}$.

Con relación a la distribución de genotipos y alelos del polimorfismo (+3954) de IL1-B, se encontró que la distribución de los genotipos estaba en equilibrio de Hardy-Weinberg (c2= 2,193, $p=0,1386$ ). Al comparar esta distribución según los grupos evaluados no hubo una diferencia estadísticamente significativa entre la presencia del polimorfismo y la RRE $(p=0,0926)$ (Fig. 3); sin embargo hubo una diferencia estadísticamente significativa cuando se comparó la distribución de alelos entre los grupos de estudio $(p=$ 0,035 ), siendo el alelo T (alelo 2 ) más prevalente en el grupo control (Tabla II).

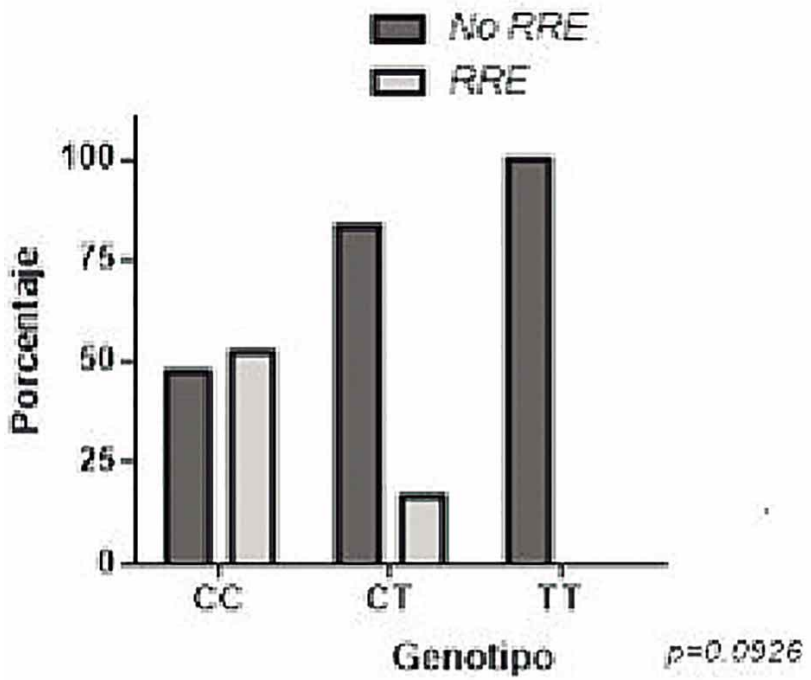

Fig. 3. Genotipos del polimorfismo IL-1B (+3954C>T). Al comparar la distribución de los genotipos no se encontró una diferencia estadísticamente significativa entre casos (RRE) y controles (NO RRE) ( $p=0.0926)$; RRE: Reabsorción radicular externa.

Tabla II. Distribución de los genotipos y alelos del polimorfismo (+3954) del gen IL-1B por grupos.

\begin{tabular}{llccccc}
\hline & & $\begin{array}{c}\text { Casos }(\mathbf{n}=\mathbf{1 2}) \\
\mathbf{n}(\%)\end{array}$ & $\begin{array}{c}\text { Controles }(\mathbf{n}=\mathbf{1 7}) \\
\mathbf{n}(\boldsymbol{\%})\end{array}$ & Valor $\boldsymbol{p}^{\mathbf{*}}$ & OR $^{\mathbf{a}}$ & IC= 95 \% \\
\hline Genotipo IL-1B & CC & $11(91,7)$ & $10(58,8)$ & & & \\
& CT & $1(8,3)$ & $5(29,4)$ & 0,1819 & 0,1818 & $0,018-1,836$ \\
\multirow{4}{*}{ Alelo } & TT & $0(0,0)$ & $2(11,8)$ & 0,4783 & 0,1826 & $0,008-4,263$ \\
& C (1) & $23(95,8)$ & $25(73,5)$ & & & \\
& T (2) & $1(4,2)$ & $9(26,5)$ & $0,0354^{*}$ & 0,1208 & $0,014-1,029$ \\
\hline
\end{tabular}

*Estadísticamente significativo, prueba exacta de Fisher; ${ }^{\circ} \mathrm{OR}$ : Odds Ratio; IC=Intervalo de confianza. 
LINCE, V. F.; DE LA OSSA, S. J.; HERNANDEZ, T. R.; BUELVAS, M. Y.; BUSTILLO, A. J. \& MADERA, A. M. Polimorfismo (+3954C>T) del gen IL-1B y su asociación con la resorción radicular apical externa post-tratamiento ortodóntico. Int. J. Odontostomat., 10(2):243-248, 2016.

\section{DISCUSIÓN}

Esta investigación tiene algunas limitaciones, entre las cuales está el tamaño de muestra, sin embargo la realización de procedimientos estandarizados y calibrados permite obtener resultados confiables, además a través de estos resultados es posible hacer estimaciones sobre la distribución genotípica y alélica del polimorfismo (+3954) del gen IL-1B en la población de estudio y su posible relación con la RRE.

Los resultados sugieren que no existe asociación estadísticamente significativa entre los genotipos del polimorfismo (+3954) de IL-1B con la presencia de RRE. Sin embargo se logró determinar que el alelo T fue más frecuente en el grupo control, lo que posiblemente puede sugerir que existen un componente genético relacionado con la presencia de RRE, lo que demuestra la necesidad de realizar otras exploraciones donde se evalúen otros tipos de polimorfismos y genes, que logren demostrar de forma veraz que la presencia de RRE posiblemente podría estar más relacionada con un factor intrínseco de tipo genético que con el tratamiento de ortodoncia. En este sentido Al-Qawasmi et al. (2003b) quien evaluó la predisposición genética a la reabsorción apical concluyó el alelo $T$ del gen en estudio puede ser considerado como alelo de bajo riesgo.

Los hallazgos del presente estudio son similares a los reportados Tomoyasu et al. (2009) quien no encontró asociación estadísticamente significativa entre el polimorfismo de IL-1B y la presencia de RRE en población Japonesa; asimismo Wu et al. (2013) a través de un metaanálisis concluyó que las variantes genotípicas (CC y CT) del polimorfismo 3954 C>T de la IL-1B no se asocia con el riesgo de RRE en comparación con los homocigotos TT, además sugirió que tampoco existen asociaciones en modelos dominantes y recesivos. Del mismo modo Linhartova et al. (2013) no logró establecer asociación entre el polimorfismo del gen IL-1B y la RRE en pacientes con ortodoncia.

Contrario a esto, autores como Iglesias-Linares et al. (2012) afirman que el desarrollo de la RRE en sujetos que se someten a un tratamiento de ortodoncia puede ser atribuible a las variaciones genéticas en el gen IL-1B; asimismo Bastos-Lages et al., encontró asociación entre el polimorfismo del gen IL-1B y la RRE en población brasilera, sugiriendo que el alelo 1 tienen mayor predisposición en sujetos con RRE. En este sentido el polimorfismo analizado en este estudio ha sido reportado como un polimorfismo funcional, es decir, que puede alterar la función del gen IL-1B, entre los modelos que se han propuesto para explicar el mecanismo mediante el cual el genotipo de IL-1B modula el grado de reabsorción radicular experimentado durante el movimiento dental ortodóntico, está el que sugiere que este polimorfismo afecta la producción de IL-1B en el caso del alelo $C$, lo que resulta en un modelado del hueso relativamente menos catabólico en la interfaz hueso cortical del ligamento periodontal a causa de la disminución del número de osteoclastos asociados con niveles más bajos de esta citoquina.

Teniendo en cuenta todos estos hallazgos se podría pensar que aún no está totalmente claro si el polimorfismo del gen IL-1B está relacionado con la presencia de RRE en individuos sometidos a tratamientos de ortodoncia, por lo cual son necesarias más exploraciones que incluyan un mayor número de variables, además otra explicación que se podría dar a los reportes contradictorios encontrados en la literatura es que los polimorfismo pueden cambiar sustancialmente dependiendo de raza o poblaciones especificas; en este sentido se ha sugerido que las poblaciones asiáticas tienen una mayor frecuencia del genotipo CC del polimorfismo IL-1B que otros grupos étnicos; del mismo modo AlQawasmi et al. (2003a) informó de que en población caucásica, los individuos con el genotipo CC del polimorfismo IL-1B tienen un alto riesgo de RRE. Asimismo, Tomoyasu et al. reportaron en población caucásica europea una frecuencia de $29,2 \%$ para el alelo $\mathrm{T}$, mientras que la población japonesa este mismo alelo tuvo una frecuencia de 5,6\%. Por otra parte, se ha sugerido que para poder aclarar estas asociaciones se debe hacer una estratificación de la población por factores intrínsecos como la etnia y factores extrínsecos tales como el consumo de tabaco, debido a que la frecuencia de muchos alelos genéticos varía entre los grupos étnicos (Greenstein \& Harte, 2002).

En conclusión el polimorfismo IL-1B (+3954C>T) se encontró presente en la población de estudio. Aunque no existieron diferencias en la distribución de los genotipos que apoyara una asociación entre este polimorfismo y la RRE, si hubo una diferencia en la distribución de los alelos, sugiriendo que el alelo T posiblemente actúa como factor protector contra el desarrollo de la RRE.

AGRADECIMIENTOS. Los autores agradecen a las directivas de la Facultad de Odontología de la Universidad de Cartagena por su apoyo para la realización de esta investigación. 
LINCE, V. F.; DE LA OSSA, S. J.; HERNANDEZ, T. R.; BUELVAS, M. Y.; BUSTILLO, A. J. \& MADERA, A. M. Polimorfismo (+3954C>T) del gen IL-1B y su asociación con la resorción radicular apical externa post-tratamiento ortodóntico. Int. J. Odontostomat., 10(2):243-248, 2016.

LINCE, V. F.; DE LA OSSA, S. J.; HERNANDEZ, T. R.; BUELVAS, M. Y.; BUSTILLO, A. J. \& MADERA, A. M. IL-1B (+3954C>T) gene polymorphism and its association with external root resorption after orthodontic treatment. Int. J. Odontostomat., 10(2):243-248, 2016.

ABSTRACT: The objective of this study was to determine the presence of Interleukin 1 beta (IL-1B) rs1143634 (+3954C>T) gene polymorphism and its association with external apical root resorption (ERR) after orthodontic treatment. We conducted a pilot study of individuals treated with orthodontic treatment, 13 (cases) had ERR after orthodontic treatment and 22 (controls) were clinically healthy. DNA was extracted from samples of epithelial cells from the oral cavity and IL-1B rs1143634 (+3954C>T) gene polymorphism was genotyped by polymerase chain reaction and digestion product through the Taql restriction enzyme. Genotype and allele frequencies of rs 1143634 were estimated; in addition, the deviation from Hardy-Weinberg equilibrium was assessed. Allele and genotype frequencies were compared using the $c 2$ test with likelihood ratio $(p<0.05)$. The mean age of participants was $28.6(S D=11.5)$ years and $68.6 \%$ were females. No statistically significant association was found between the genotypes distribution of IL-1B (+3954C>T) polymorphism with ERR ( $p=0.0926)$. However, a significant difference in the alleles distribution $(p=0.035)$ was observed, where the allele T (allele 2$)$ was more prevalent in the control group. IL-1B (+3954C>T) polymorphism was present in the study population. Although there were no differences in the genotypes distribution to support an association between this polymorphism with ERR, there was a difference in the alleles distribution, suggesting that the allele T possibly acts as a protective factor against the development of ERR.

\section{KEY WORDS: external root resorption, apical resorption, Interleukin-1 beta.}

\section{REFERENCIAS BIBLIOGRÁFICAS}

Ahangari, Z.; Nasser, M.; Mahdian, M.; Fedorowicz, Z. \& Marchesan, M. A. Interventions for the management of external root resorption. Cochrane Database Syst. Rev., (6):CD008003, 2010.

Al-Qawasmi, R. A.; Hartsfield, J. K. Jr.; Everett, E. T.; Flury, L.; Liu, L.; Foroud, T. M.; Macri, J. V. \& Roberts, W. E. Genetic predisposition to external apical root resorption in orthodontic patients: linkage of chromosome-18 marker. J. Dent. Res., 82(5):356-60, 2003a.

Al-Qawasmi, R. A.; Hartsfield, J. K. Jr.; Everett, E. T.; Flury, L.; Liu, L.; Foroud, T. M. \& Roberts, W. E. Genetic predisposition to external apical root resorption. Am. J. Orthod. Dentofacial Orthop., 123(3):242-52, 2003b.

Bansal, P.; Nikhil, V. \& Kapur, S. Multiple idiopathic external apical root resorption: A rare case report. J. Conserv. Dent., 18(1):70-2, 2015.

Bastos, J. V.; Côrtes, M. I.; Silva, J. F.; Goulart, E. M.; Colosimo, E. A.; Gomez, R. S. \& Dutra, W. O. A study of the interleukin-1 gene cluster polymorphisms and inflammatory external root resorption in replanted permanent teeth. Int. Endod. J., 48(9):878-87, 2015.

Bastos Lages, E. M.; Drummond, A. F.; Pretti, H.; Costa, F. O.; Lages, E. J.; Gontijo, A. I.; Miranda Cota, L. O. \& Brito, R. B. Jr. Association of functional gene polymorphism IL-1 beta in patients with external apical root resorption. Am. J. Orthod. Dentofacial Orthop., 136(4):542-6, 2009.

Baysal, A.; Uysal, T.; Ozdamar, S.; Kurt, B.; Kurt, G. \& Gunhan, O. Comparisons of the effects of systemic administration of L-thyroxine and doxycycline on orthodontically induced root resorption in rats. Eur. J. Orthod., 32(5):496-504, 2010.

Diouf, J. S.; Benoist, F. L. \& Benoist, H. M. External inflammatory root resorption associated with a traumatic occlusion. J. Clin. Orthod., 49(3):195-200, 2015.

Gonzales, C.; Hotokezaka, H.; Karadeniz, E. I.; Miyazaki, T.; Kobayashi, E.; Darendeliler, M. A. \& Yoshida, N. Effects of fluoride intake on orthodontic tooth movement and orthodontically induced root resorption. Am. J. Orthod. Dentofacial Orthop., 139(2):196-205, 2011.

Greenstein, G. \& Hart, T. C. A critical assessment of interleukin-1 (IL-1) genotyping when used in a genetic susceptibility test for severe chronic periodontitis. J. Periodontol., 73(2):231-47, 2002.
Iglesias-Linares, A.; Yañez-Vico, R. M.; Ortiz-Ariza, E.; Ballesta, S.; Mendoza-Mendoza, A.; Perea, E. \& Solano-Reina, E. Postorthodontic external root resorption in root-filled teeth is influenced by interleukin1b polymorphism. J. Endod., 38(3):283-7, 2012.

Linhartova, P.; Cernochova, P. \& Izakovicova Holla, L. IL1 gene polymorphisms in relation to external apical root resorption concurrent with orthodontia. Oral Dis., 19(3):262-70, 2013.

Sharab, L. Y.; Morford, L. A.; Dempsey, J.; Falcão-Alencar, G.; Mason, A.; Jacobson, E.; Kluemper, G. T.; Macri, J. V. \& Hartsfield, J. K. Jr. Genetic and treatment-related risk factors associated with external apical root resorption (EARR) concurrent with orthodontia. Orthod. Craniofac. Res., 18 (Suppl. 1):71-82, 2015.

Tomoyasu, Y.; Yamaguchi, T.; Tajima, A.; Inoue, I. \& Maki, K. External apical root resorption and the interleukin-1B gene polymorphism in the Japanese population. Orthod. Waves, 68(4):152-7, 2009

Villa, P. A.; Oberti, G.; Moncada, C. A; Vasseur, O.; Jaramillo, A.; Tobón, D. \& Agudelo, J. A. Pulp-dentine complex changes and root resorption during intrusive orthodontic tooth movement in patients prescribed nabumetone. J. Endod., 31(1):61-6, 2005

Wu, F. L.; Wang, L. Y.; Huang, Y. Q.; Guo, W. B.; Liu, C. D. \& Li, S. G. Interleukin-1b +3954 polymorphisms and risk of external apical root resorption in orthodontic treatment: a meta-analysis. Genet. Mol. Res., 12(4):4678-86, 2013.

Dirección para Correspodencia:

Meisser Vidal Madera Anaya

Universidad de Cartagena

Campus Ciencias de la Salud

Facultad de Odontología

Departamento de Investigaciones

Cartagena - Bolívar - COLOMBIA

Email: mmaderaa@unicartagena.edu.co 